\section{Editorial Policies and Procedures}

The mission of the Animal Genetic Resources Information Bulletin (AGRI) is the promotion of information on the better use of animal genetic resources of interest to food and agriculture production, under the Global Strategy for the Management of Farm Animal Genetic Resources. All aspects of the characterization, conservation and utilization of these resources are included, in accordance with the Convention on Biological Diversity. AGRI will highlight information on the genetic, phenotypic and economic surveying and comparative description, use, development and maintenance of animal genetic resources; and on the development of operational strategies and procedures which enable their more cost-effective management. In doing this AGRI will give special attention to contributions dealing with breeds and procedures capable of contributing to the sustainable intensification of the world's medium to low input production environments (agro-ecosystems), which account for the substantial majority of the land area involved in livestock production; the total production of food and agriculture from livestock; and of our remaining farm animal genetic resources.

Views expressed in the paper published in AGRI represent the opinions of the author(s) and do not necessarily reflect those of the institutions which the authors are affiliated, FAO or the Editors.

The suitability of manuscripts for publication in AGRI is judged by the Editors and reviewers.

\section{Electronic publication}

AGRI is available in full electronically on the Internet, in addition to being published in hard copy, at: << http://www.fao.org/dad-is >>

\section{Types of Articles}

The following types of articles are published in AGRI.

\section{Research articles}

Findings of work on characterization, conservation and utilization of farm animal genetic resources (AnGR) in well described production environments, will be considered for publication in AGRI. Quality photographs of these genetic resources viewed in the primary production environment to which they are adapted, accompanying the manuscripts are encouraged.

\section{Review articles}

Unsolicited articles reviewing agro-ecosystems, country-level, regional or global developments on one or more aspects of the management of animal genetic resources, including state-of-the-art review articles on specific fields in AnGR, will be considered for publication in AGRI.

\section{Position papers}

Solicited papers on topical issues will also be published as deemed required.

\section{Other published material}

This includes book reviews, news and notes covering relevant meetings, training courses and major national, regional and international events and conclusions and recommendations associated with the outcomes of these major events. Readers are encouraged to send such items to the editors.

\section{Guidelines for Authors}

\section{Manuscript submission}

Manuscripts prepared in English, French or Spanish with an English summary and 
another summary in either French or Spanish, should be submitted to AGRI Editor, AGAP, FAO, Viale delle Terme di Caracalla, 00100 Rome, Italy. Alternatively a manuscript may be sent as a WinWord Electronic Mail attachment to < agri@fao.org > . Photographs, coloured or black and white, and figures must be always sent by mail.

Manuscripts should be typed double-spaced and with lines numbered in the left margin. All pages, including those of references, tables etc., must be consecutively numbered. The corresponding author is notified of the receipt of a manuscript.

For manuscripts that are accepted after revision, authors are encouraged to submit a last version ( $3 \frac{1}{2} 2^{\prime \prime}$ disc format) in Word 6.0 for Windows of their revised manuscript along with the printed copy.

\section{Preparation of the manuscript}

The first page of the manuscript must include the running head (abbreviated title), title, names of authors, institutions, full addresses including postal codes and telephone number and other communication details (fax, e-mail, etc.) of the corresponding author. The running head not exceeding 45 characters plus spaces, should appear at the top of page 1 of the manuscript entirely in capital letters. The title of the manuscript is typed in upper and lower case letters. The title should be as brief as possible not exceeding 150 characters (including spaces) with species names when applicable. Authors, institutions and addresses are in upper and lower case italics. There is one blank line between the title and the authors. Addresses are typed as footnotes to the authors after leaving one blank line. Footnotes are designated numerically. Two lines are left below the footnotes.

\section{Headings}

Headings of sections, for example Summary, Introduction, etc., are left-justified. Leave two blank lines between addresses footnotes and Summary and between the heading Summary and its text. Summary should not exceed 200 words . It should be an objective summary briefly describing the procedures and findings and not simply stating that the study was carried on such and such and results are presented, etc. Leave one line between the summary text and Keywords which is written in italics as well as the keywords themselves. All headings of sections (14 regular) and sub-sections (12 regular) are typed bold and preceded and succeeded by one blank line and their text begins with no indention. The heading of a sub-subsection is written in italics, and ends with a dot after which the text follows on the same line. Keywords come immediately after the summaries. They should be no more than six, with no "and" or "\&".

\section{Tables and figures}

Tables and figures must be enclosed with the paper and attached at the end of the text according their citation in the document. Photos will not be returned

\section{Tables}

Tables, including footnotes, should be preceded and succeeded by 2 blank lines. Table number and caption are written, above the table, in italics (12) followed by a dot, then one blank line. For each column or line title or sub-title, only the 1st letter of the 1st word is capitalized. Tables should be numbered consecutively in Arabic numerals. Tables and captions should be left justified as is the text. Use horizontal or vertical lines only when necessary. Do not use tabs or space-bar to create a table but only the appropriate commands.

\section{Figures}

Figures including titles and legends should be preceded and succeeded by two blank lines. Figure number and title are written, below the figure, in italics (12) and end with a dot. The term figures includes photos, line drawings, maps, diagrams etc.

All the submitted diagrams, must be 
accompanied with the original matrix of the data used to create them. It is strongly advised to submit diagrams in Word 6.0 or Excel 5.0. Figures should be numbered consecutively in Arabic numerals.

\section{References}

Every reference cited in the text should be included in the reference list and every reference in the reference list should have been mentioned in the text at least once. References should be ordered firstly alphabetically by the first author's surname and secondly by year.

Example for reference in a periodical is:

Köhler-Rollefson, I., 1992; The camel breeds of India in social and historical perspective. Animal Genetic Resources Information 10, 53-64.

When there are more than one author:

Matos, C.A.P., D.L. Thomas, D. Gianola, R.J. Tempelman \& L.D. Young, 1997; Genetic analysis of discrete reproductive traits in sheep using linear and nonnlinear models: 1 . Estimation of genetic parameters 75, 76-87.
For a book or an ad hoc publication, e.g., reports, theses, etc.:

Cockril, W.R., (Ed), 1994; The Husbandry and Health of the Domestic Buffalo. FAO, Rome, Italy, pp 993.

For an article in the proceedings of a meeting:

Hammond, K., 1996; FAO's programme for the management of farm animal genetic resources. In C. Devendra (Ed.) Proceedings of IGA/FAO Round Table on the Global Management of Small Ruminant Genetic Resources, Beijing, May 1996, FAO, Bangkok, Thailand, 4-13.

Where information included in the article has been obtained or derived from a World Wide Web site, then quote in the text, e.g. "derived from FAO. 1996" and in the References quote the URL standard form: FAO, 1996; Domestic Animal Diversity Information System <http:/ / www.fao.org/ dad-is/>, FAO, Rome 


\section{Normes et règles éditoriales}

L'objectif du Bulletin d'Information sur les Ressources Génétiques Animales (AGRI) est la vulgarisation de l'information disponible sur la meilleure gestion des ressources génétiques animales d'intérêt pour la production alimentaire et agricole, d'après les recommendations de la Stratégie Mondiale pour la Gestion des Ressources Génétiques des Animaux Domestiques. Tous les aspects relatifs à la caractérisation, la conservation et l'utilisation de ces ressources seront pris en considération, suivant les normes de la Convention pour la Biodiversité.

AGRI désire diffuser de l'information sur la génétique, les enquêtes phénotypiques et économiques et les desciptions comparatives, l'utilisation et la conservation des ressources génétiques animales, ainsi que toute information sur le développement de stratégies opérationnelles et de normes qui puissent permettre une meilleure gestion de la relation coût/efficacité. C'est pour cela que AGRI prendra spécialement en considération toutes les contributions référées aux races et aux normes capables de permettre une intensification durable des milieux (agroécosystèmes) à revenus moyens et bas dans le monde; qui comprennent la majeur partie des terres consacrées à l'élevage, à la production totale des aliments et l'agriculture provenants de l'élevage; et tout ce qui reste comme ressources génétiques des animaux domestiques.

Les opinions exprimées dans les articles publiés dans AGRI appartiennent seulement aux auteurs et donc ne représentent pas nécessairement l'opinion des instituts pour lesquels ils travaillent, la FAO ou les éditeurs.

L'opportunité ou non de publier un article dans AGRI sera jugée par les éditeurs et les réviseurs.

\section{Publication électronique}

En plus de sa version imprimée, la version totale de AGRI se trouve disponible sur Internet, sur le site: <<http://www.fao.org/dad-is/>>

\section{Types d'articles}

Les articles suivants pourront être publiés sur AGRI:

\section{Articles de recherche}

Seront prises en considération pour leur publication sur AGRI les études sur la caractérisation, la conservation et l'utilisation des ressources génétiques des animaux domestiques (AnGR) accompagnées d'une bonne description du milieu. On encourage les auteurs à envoyer des photographies de bonne qualité qui montrent les races en question dans leur milieu naturel de production.

\section{Révisions}

Occasionnellement, des articles contenant une révision des agroécosystèmes, au niveau national, régional ou mondial, avec un ou plusieurs aspects se rapportant à la gestion des ressources génétiques animales, y comprises les mises à jour des différentes zones de AnGR, seront pris en considération.

\section{Articles spécifiques}

Ponctuellement, des articles sur des thèmes spécifiques pourront être demandés pour la publication d'éditions spéciales.

\section{Autre matériel pour publication}

Ceci comprend la révision de livres, nouvelles et notes de réunions importantes, cours de formation et principaux évènements nationaux, régionaux et internationaux; ainsi que les conclusions et recommendations par rapport aux objectifs des ces principaux évènements. Les auteurs sont priés d'envoyer ce genre de matériel aux éditeurs. 


\section{Guide pour les auteurs}

\section{Présentation du manuscript}

Les articles se présenteront en anglais, français ou espagnol, avec un résumé en anglais et sa traduction en français ou en espagnol; et seront envoyés à l'éditeur de AGRI, AGAP, FAO, Viale delle Terme di Caracalla, 00100 Rome, Italie. L'autre possibilité est d'envoyer l'article par courrier électronique avec le document adjoint en version WinWord à <agri@fao.org>. Les photographies, en couleur ou en blanc et noir, seront toujours envoyées par courrier normal.

Les manuscripts se présenteront à double interligne et avec le numéro correspondant à chaque ligne sur la marge gauche. Toutes les pages seront numérotées, y comprises celles avec les références bibliographiques, les tableaux, etc. L'auteur recevra une lettre lui donnant bonne réception de son document.

Lorsqu'un article, après sa révision, sera accepté, on demandera à l'auteur d'envoyer la version finale révisée sur disquette (format $31 / 2^{\prime \prime}$ ) en Word $6.0 \times$ Windows, ainsi qu'une copie sur papier.

\section{Préparation du manuscript}

Sur la première page du manuscript on indiquera le titre de l'article en abrégé, le titre et noms des auteurs, des institutions, les adresses complètes ( $\mathrm{y}$ compris code postal et numéro de téléphone); ainsi que tout autre moyen de contact tel que fax, e-mail, etc. avec l'auteur principal. Le titre abrégé ne devra pas dépasser les 45 caractères, plus les espaces nécessaires, et s'écrira sur la partie supérieure de la page $1 \mathrm{du}$ manuscript en majuscules. Le titre en entier du manuscript sera écrit en majuscules et minuscules; il devra être aussi bref que possible, sans dépasser les 150 caractères (y compris les espaces nécessaires), et avec l'indication des noms des espèces. Les noms des auteurs, des institutions et les adresses seront en italique et en lettres majuscules et minuscules. On laissera un espace en blanc entre le titre et les noms des auteurs. Les adresses seront indiquées comme des notes à pied de page pour chacun des auteurs après avoir laissé un espace en blanc après les noms. Chaque note de pied de page sera numérotée. On laissera deux espaces en blanc après les adresses.

\section{Titres}

Les titres de chaque chapitre, par example Résumé, Introduction, etc. seront alignés à gauche. Laisser deux espaces en blanc entre les notes de pied de page avec les adresses et le Résumé, et entre le titre Résumé et le texte qui suit. Le résumé ne devra pas dépasser les 200 mots. Il s'agira d'un résumé objectif qui fasse une brève description des processus utilisés et des résultats obtenus, et non pas une simple présentation du travail réalisé avec une description générale des résultats. Laisser un espace en blanc entre la fin du texte du résumé et les mots-clés, qui seront écrits en italique ainsi que le titre Mots-clés. Les mots-clés seront au maximum six et il ne devra pas y avoir de "et" ou "\&". Tous les titres principaux de chapitre (14 regular) et sous-chapitre (12 regular) seront en gras avec un espace en blanc avant et après. Le texte commencera sans retrait. Un titre à l'intérieur d'un sous-chapitre s'écrira en italique, suivi $\mathrm{d}^{\prime}$ un point, avec le texte à continuation.

\section{Tableaux et figures}

Les tableaux et les figures iront à la fin du texte en suivant l'ordre d'apparition dans le texte. Les photographies ne seront pas dévolues aux auteurs.

\section{Tableaux}

Les tableaux, y compris les notes de pied de page, devront avoir un espace en blanc avant et après. Le numéro du tableau et le titre s'écriront sur la partie supérieure en italique (12) avec un point à la fin et un espace en blanc en dessous. Sur chaque colonne, titre d'en-tête ou sous-titre, seulement la première lettre du premier mot sera en majuscule. Les tableaux et leur titre seront alignés à gauche, ainsi que le texte. Les lignes verticales et 
horizontales seront utilisées seulement si nécessaires. Ne pas utiliser les tabs ou la barre de séparation pour créer un tableau.

\section{Figures}

Les figures, y compris les titres et les légendes, seront précédés et suivis de deux espaces en blanc. Le numéro de la figure et le titre s'écriront sur la partie supérieure en italique (12) avec un point à la fin. Sous la rubrique figure on trouvera les photographies, les graphiques, les cartes, les diagrammes, etc. Dans le cas des diagrammes, la matrice originale avec les données utilisées pour son élaboration devra être envoyée. On recommande l'utilisation de Word 6.0 ou Excel 5.0 pour la présentation des diagrammes.

\section{Références}

Toute référence présente dans le texte devra apparaître sur la liste des références, et chaque référence de la liste aura été citée au moins une fois dans le texte. Les références iront en ordre alphabétique du nom de l'auteur, suivi de l'année. Example dans le cas d'une référence sur une revue:

Köhler-Rollefson, I.,1992; The camel breeds of India in social and historical perspective. Animal Genetic Resources Information 10, 53-64.
Lorsqu'il s'agit de plus d'un auteur:

Matos, C.A.P., D.L. Thomas, D. Gianola, R.J. Tempelman \& L.D. Young, 1997; Genetic analysis of discrete reproductive traits in sheep using linear and nonnlinear models: 1. Estimation of genetic parameters 75, 76-87.

Dans le cas d'un livre ou d'une publication ad hoc, par example un rapport, une thèse, etc.:

Cockril, W.R., (Ed), 1994; The Husbandry and Health of the Domestic Buffalo. FAO, Rome, Italy, pp 993.

S'il s'agit d'un acte d'une réunion:

Hammond, K., 1996; FAO's programme for the management of farm animal genetic resources. In C. Devendra (Ed.) Proceedings of IGA/FAO Round Table on the Global Management of Small Ruminant Genetic Resources, Beijing, May 1996, FAO, Bangkok, Thailand, 4-13.

Lorsque l'information contenue dans l'article ait été obtenue ou dérive d'un site World Wide Web, il faudra mettre le texte entre guillemets; par example "tiré de la FAO. 1996" et indiquer dans les Références la forme standard URL:

FAO, 1996; Domestic Animal Diversity Information System <http://www.fao.org/ dad-is/>, FAO, Rome 


\section{Reglas y normas editoriales}

El objetivo del Boletín de Información sobre Recursos Genéticos Animales (AGRI) es la divulgación de la información sobre una mejor gestión de los recursos genéticos animales de interés para la producción alimentaria y agrícola, siguiendo la Estrategia Mundial para la Gestión de los Recursos Genéticos de los Animales Domésticos. Todos los aspectos referidos a la caracterización, la conservación y el uso de estos recursos serán tomados en consideración, de acuerdo con la Convención sobre la Biodiversidad.

AGRI publicará información sobre genética, encuestas fenotípicas y económicas y descripciones comparativas, uso, desarrollo y conservación de los recursos genéticos animales, así como sobre el desarrollo de estrategias operacionales y normas que permitan una gestión más eficaz de la relación costo/eficacia. Por ello, AGRI prestará especial atención a las contribuciones referidas a razas y normas capaces de contribuir a la intensificación sostenible de los medios (agroecosistemas) con ingresos medio y bajos en el mundo, que comprenden casi la mayor parte de las tierras dedicadas a la producción ganadera; la producción total de alimentos y agricultura provenientes de la ganadería; y el resto de los recursos genéticos de animales domésticos.

Los puntos de vista expresados en los artículos publicados en AGRI son solamente las opiniones de los autores y, por tanto, no reflejan necesariamente la opinión de las instituciones para las cuales trabajan dichos autores, de la FAO o de los editores.

La oportunidad o no de publicar un artículo en AGRI será juzgada por los editores y revisores.

\section{Publicación electrónica}

Además de su publicación impresa, la versión íntegra de AGRI se encuentra disponible electrónicamente sobre Internet, en el sito: <<http://www.fao.org/dad-is/>>

\section{Tipos de artículos}

Serán publicados en AGRI los siguientes tipos de artículos:

\section{Artículos sobre investigación}

Se tomarán en consideración para su publicación en AGRI los estudios sobre la caracterización, conservación y uso de los recursos genéticos de los animales domésticos (AnGR) con una buena descripción del entorno. Se agradecerá el envío de fotografías de calidad que presenten a las razas en cuestión en su ambiente natural de producción.

\section{Artículos de revisión}

Se podrán tener en consideración ocasionalmente aquellos artículos que presenten una revisión de los agroecosistemas, a nivel nacional, regional o mundial, con el desarrollo de uno o más aspectos referidos a la gestión de los recursos genéticos animales, incluidas las revisiones sobre el estado actual de las distintas áreas de AnGR.

\section{Artículos específicos}

Se solicitarán puntualmente artículos sobre temas específicos para ediciones especiales.

\section{Otro material para publicación}

Incluye la revisión de libros, noticias y notas referidas a reuniones importantes, cursos de formación y principales eventos nacionales, regionales e internacionales, así como conclusiones y recomendaciones relacionadas con los objetivos de estos principales eventos. Se invita a los lectores a enviar este tipo de material a los editores. 


\section{Guía para los autores}

\section{Presentación del manuscrito}

Los artículos se presentarán en inglés, francés o español, junto con un resumen en inglés y su traducción en francés o español, y se enviarán al editor de AGRI, AGAP, FAO, Viale delle Terme di Caracalla, 00100 Roma, Italia. Otra posibilidad es enviar el artículo por correo electrónico adjuntando el documento en versión WinWord a <agri@fao.org>. Las fotografías, a color o en blanco y negro, se enviarán siempre por correo normal.

Los manuscritos se presentarán con doble espacio y con el número correspondiente a cada línea en el margen izquierdo. Todas las páginas serán numeradas, incluidas las de las referencias bibliográficas, cuadros, etc. El autor recibirá una notificación sobre la recepción de su documento.

En el caso de aceptación de un artículo después de su revisión, se solicitará al autor una versión final de su artículo revisado en disquete (formato $31 / 2^{\prime \prime}$ ) en Word $6.0 \mathrm{x}$ Windows, así como una copia impresa del mismo.

\section{Preparación del manuscrito}

En la primera página del manuscrito se indicará el título abreviado del artículo, títulos y nombres de los autores, instituciones, direcciones completas (incluido código postal y número de teléfono); así como otros medios de contacto tales como fax, e-mail, etc., del autor principal. El título abreviado no deberá sobrepasar los 45 caracteres más los espacios correspondientes, y aparecerá en la parte superior de la página 1 del manuscrito en mayúsculas. El título entero del manuscrito viene escrito en mayúsculas y minúsculas. Dicho título debe ser lo más breve posible y no sobrepasar los 150 caracteres (incluidos los espacios necesarios), con los nombres de las especies, si necesario. Los nombres de los autores, instituciones y direcciones se escribirán en cursiva y en letras mayúsculas y minúsculas. Se dejará una línea en blanco entre el título y los nombres de los autores. Las direcciones se escribirán como notas de pie de página de cada autor después de dejar una línea en blanco entre los nombres y éstas. Cada nota de pie de página con la dirección vendrá indicada numéricamente. Se dejarán dos líneas en blanco después de las direcciones.

\section{Títulos}

Los títulos de cada sección, por ejemplo Resumen, Introducción, etc., vienen alineados a la izquierda. Dejar dos líneas en blanco entre las notas de pie de página con las direcciones y el Resumen y entre el título Resumen y el texto que sigue. El resumen no deberá exceder de 200 palabras. Deberá ser un resumen objetivo que describa brevemente los procesos y logros obtenidos, y no una presentación de cómo se ha llevado a cabo el estudio y una descripción genérica de los resultados. Dejar una línea en blanco entre el final del texto del resumen y las palabras clave, que se escribirán en cursiva así como el titulo Palabras clave. No deberán ser más de seis y no deberán contener " $y$ " $\mathrm{o}$ " $\&$ ". Todos los títulos principales de capítulo (14 regular) y subcapítulo (12 regular) serán en negrita e irán precedidos y seguidos de una línea en blanco. El texto correspondiente empezará sin sangrado. Un título dentro de un subcapítulo se escribirá en cursiva e ira seguido de un punto con a continuación el texto correspondiente.

\section{Cuadros y figuras}

Los cuadros y las figuras se incluirán al final del texto siguiendo el orden de cita dentro del mismo. Las fotografías no serán devueltas a sus autores.

\section{Cuadros}

Los cuadros, incluidas las notas de pie de página, deberán ir precedidos y seguidos por dos líneas en blanco. El numero del cuadro y su título se escribirán en la parte superior en cursiva (12) con un punto al final y seguido 
de una línea en blanco. En cada columna o título de encabezamiento o subtítulo, sólo la primera letra de la primera palabra irá en mayúscula. Los cuadros irán numerados de forma consecutiva con números árabes. Los cuadros y sus títulos se alinearán a la izquierda, así como el texto. Se utilizarán líneas horizontales o verticales sólo cuando sea necesario. No utilizar tabuladores o la barra espaciadora para crear un cuadro.

\section{Figuras}

Las figuras, incluidos los títulos y leyendas, irán precedidas y seguidas de dos líneas en blanco. El número de la figura y el título se escribirán en la parte superior en cursiva (12) con un punto al final. La palabra figura incluye las fotografías, los gráficos, los mapas, los diagramas, etc. En el caso del diagrama se enviará la matriz original con los datos utilizados para crearlo. Se recomienda encarecidamente la utilización de Word 6.0 o Excel 5.0 para la presentación de los diagramas.

\section{Referencias}

Toda referencia presente en el texto deberá aparecer en la lista de referencias y, de la misma manera, cada referencia de la lista deberá haber sido citada por lo menos una vez en el texto. Las referencias deben ir en orden alfabético del apellido del autor, seguido por el año.
Ejemplo en el caso de una referencia de una revista:

Köhler-Rollefson, I.,1992; The camel breeds of India in social and historical perspective. Animal Genetic Resources Information 10, 53-64.

Cuando se trata de más de un autor:

Matos, C.A.P., D.L. Thomas, D. Gianola, R.J. Tempelman \& L.D. Young, 1997; Genetic analysis of discrete reproductive traits in sheep using linear and nonnlinear models: 1. Estimation of genetic parameters 75, 76-87.

En el caso de un libro o de una publicación ad hoc, por ejemplo informes, tesis, etc.:

Cockril, W.R., (Ed), 1994; The Husbandry and Health of the Domestic Buffalo. FAO, Rome, Italy, pp 993.

Cuando se trate de un artículo dentro de las actas de una reunión:

Hammond, K., 1996; FAO's programme for the management of farm animal genetic resources. In C. Devendra (Ed.) Proceedings of IGA/FAO Round Table on the Global Management of Small Ruminant Genetic Resources, Beijing, May 1996, FAO, Bangkok, Thailand, 4-13.

Cuando la información contenida en el artículo haya sido obtenida o derive de un sito World Wide Web, poner el texto entre comillas; por ejemplo "sacado de la FAO. 1996" e indicar en las Referencias la forma estándar URL:

FAO, 1996; Domestic Animal Diversity Information System <http://www.fao.org/ dad-is/>, FAO, Rome 